\title{
Harmonic Chirp Imaging Method for Ultrasound Contrast Agent
}

\author{
Jerome M. G. Borsboom, Member, IEEE, Chien Ting Chin, Member, IEEE, \\ Ayache Bouakaz, Member, IEEE, Michel Versluis, and Nico de Jong, Associate Member, IEEE
}

\begin{abstract}
Coded excitation is currently used in medical ultrasound to increase signal-to-noise ratio (SNR) and penetration depth. We propose a chirp excitation method for contrast agents using the second harmonic component of the response. This method is based on a compression filter that selectively compresses and extracts the second harmonic component from the received echo signal. Simulations have shown a clear increase in response for chirp excitation over pulse excitation with the same peak amplitude. This was confirmed by two-dimensional (2-D) optical observations of bubble response with a fast framing camera. To evaluate the harmonic compression method, we applied it to simulated bubble echoes, to measured propagation harmonics, and to B-mode scans of a flow phantom and compared it to regular pulse excitation imaging. An increase of approximately $10 \mathrm{~dB}$ in SNR was found for chirp excitation. The compression method was found to perform well in terms of resolution. Axial resolution was in all cases within $10 \%$ of the axial resolution from pulse excitation. Range side-lobe levels were $30 \mathrm{~dB}$ below the main lobe for the simulated bubble echoes and measured propagation harmonics. However, side-lobes were visible in the B-mode contrast images.
\end{abstract}

\section{INTRODUCTION}

$I^{\mathrm{N}}$ $\mathrm{N}$ the last decade, several new pulsing schemes and signal processing methods were developed for ultrasound contrast imaging. Regular diagnostic imaging saw the advent of harmonic imaging and multipulse excitation schemes such as pulse inversion and power modulation [1][3]. These methods, which rely on selective extraction of spectral components in the received echo, have been shown to provide significant improvements in image quality and contrast-to-tissue ratio (CTR). More recently, coded excitation was introduced. Coded excitation operates by transmission of long pulses in which a clearly recognizable signature, the code, is embedded [4]. After reception, the resulting echo signal is filtered through an autocorrelation-based

Manuscript received December 29, 2003; accepted July 21, 2004. This work was supported by Technology Foundation STW (RKG.5104)

J. M. G. Borsboom, A. Bouakaz, and N. de Jong are with the Department of Experimental Echocardiography, Thoraxcentre, Erasmus MC, Rotterdam, The Netherlands (e-mail: j.borsboom@erasmusmc.nl). They also are with the Interuniversitary Cardiology Institute of the Netherlands (ICIN), Utrecht, The Netherlands.

At the time of this research, C. T. Chin was with the Department of Experimental Echocardiography, Thoraxcentre, Erasmus MC, Rotterdam, The Netherlands, and with the Foundation for Fundamental Research on Matter (FOM), Utrecht, The Netherlands. C. T. Chin is now with Philips Research, Briarcliff Manor, NY.

M. Versluis and N. de Jong are with Physics of Fluids, University of Twente, Enschede, The Netherlands. filter to detect and remove the code, a process called decoding or compression. A good code is well detectable in the received echo and encompasses more bandwidth than an equal length Gaussian pulse. At compression time, this bandwidth is used to obtain good range resolution. As the axial resolution after decoding depends on the bandwidth of the excitation signal, images with good axial resolution can be obtained using long pulses combined with coded excitation. However, range side lobes may result from decoding due to a partial match of the code and the compression filter for certain time shifts.

Codes suitable for ultrasound applications can be divided into two categories based on the type of coding [5]. One category consists of codes based on phase-modulated signals. In these codes a long sinusoidal burst of constant frequency is modulated by alternating the phase of subsequent parts of the sinusoid over a fixed set of phase values. For example, for binary codes such as Barker codes and Golay codes, the phase alternates between $0^{\circ}$ and $180^{\circ}$. The other category consists of codes based on frequency modulation. In these codes the instantaneous frequency of a long sinusoidal burst is modulated over time. For ultrasound imaging, the most important code in this category is the linear frequency sweep or linear chirp as it is relatively robust to frequency shifts. In general, a shift in mean frequency is found in signals received from frequencydependent attenuating media such as tissue. This attenuation, which is approximately proportional to frequency, causes the chirp to be shifted downward in frequency. However, as the frequency shift translates after decoding into a time shift, the response from an attenuating medium will be slightly shifted only in time, which is acceptable for imaging purposes [5]. A linear chirp is a long sinusoidal burst with an instantaneous frequency changing linearly in time. The amplitude may be apodized to suppress range side-lobe generation from the decoding filter [6]. Conventionally, chirps have been used to increase the signal-tonoise ratio (SNR) when peak transmission amplitude is limited. Improvements in SNR of more than $10 \mathrm{~dB}$ have been reported [7].

Currently available ultrasound contrast agents (UCA) consist of small (1-10 $\mu \mathrm{m}$ diameter), gas-filled, encapsulated micro spheres. Like free air bubbles, contrast agent bubbles are strong ultrasound scatterers, both linearly and nonlinearly. Strong nonlinear scattering from contrast agents enables discriminatory detection in tissues, which is used in several nonlinear contrast agent imaging methods. The performance of these methods is dependent on 


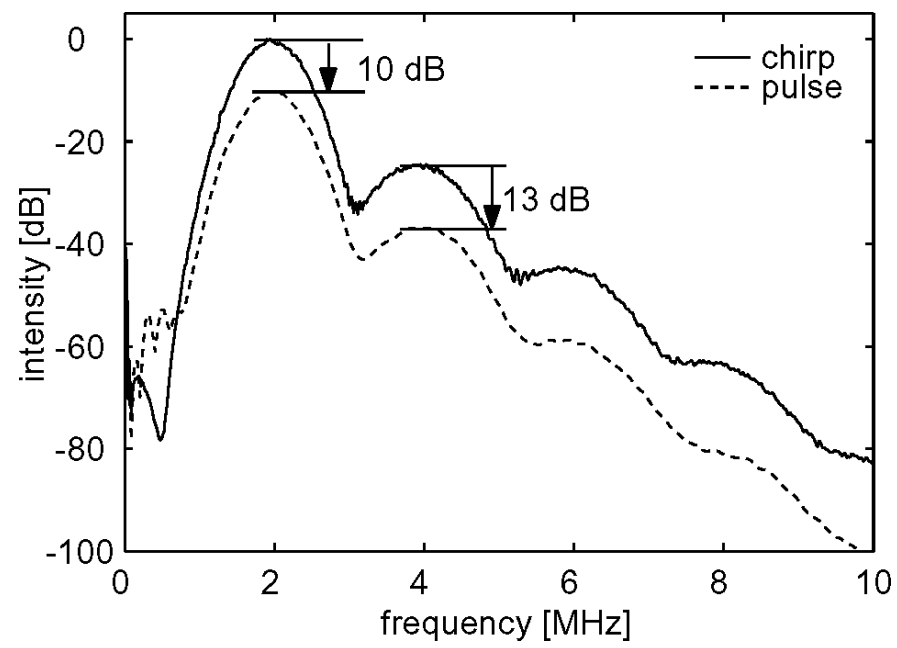

Fig. 1. Simulated response of 7000 contrast agent bubbles randomly distributed in space with SonoVue size distribution excited with chirp $(-)$ and pulse (---) with equal center frequency $(2 \mathrm{MHz})$, bandwidth $(45 \%)$, and peak amplitude $(50 \mathrm{kPa})$.

the relative contributions of the nonlinear response from contrast agent and surrounding tissue that is quantified in the CTR. Important application areas for UCA are the enhancement of hypo-echoic regions, perfusion imaging, and functional assessment of, for example, the myocardium [8].

In nondestructive contrast agent imaging, the peak transmitted acoustic pressure is limited by the destruction threshold of the contrast agent. This threshold is usually much lower than the maximum allowed mechanical index (MI), which limits the peak transmitted pressure to avoid cavitation and tissue damage [9]. Therefore, nondestructive contrast agent imaging is more severely limited in transmitted peak amplitude and produces images with lower SNR than conventional imaging and tissue harmonic imaging. To improve the SNR in contrast agent imaging, one can either lower the noise level or increase the signal level. The noise level is mainly fixed by system design. Increasing the signal level by increasing the transmission amplitude is limited by the bubble destruction threshold. Coded excitation can be applied to increase the signal energy without increasing the peak transmitted amplitude by using longer pulses and compression on receive.

Previously, we reported [10] in a simulation study which showed that chirp excitation can increase the relative level of second harmonic over pulse excitation, which potentially results in improved CTR. The main result from the study of [10] is summarized in Fig. 1, which shows in frequency domain the simulated response of 7000 bubbles randomly distributed in space and with SonoVue ${ }^{\mathrm{TM}}$ (Bracco Research SA, Geneva, Switzerland) size distribution to a pulse and a chirp having equal center frequency (2 MHz), bandwidth (45\%), and peak amplitude (50 kPa). It is clear that, apart from an energy difference of $10 \mathrm{~dB}$ between pulse and chirp - which explains the difference between the curves at the fundamental - there is an additional difference of approximately $3 \mathrm{~dB}$ at the second harmonic. This effect reflects the increased radial excur-

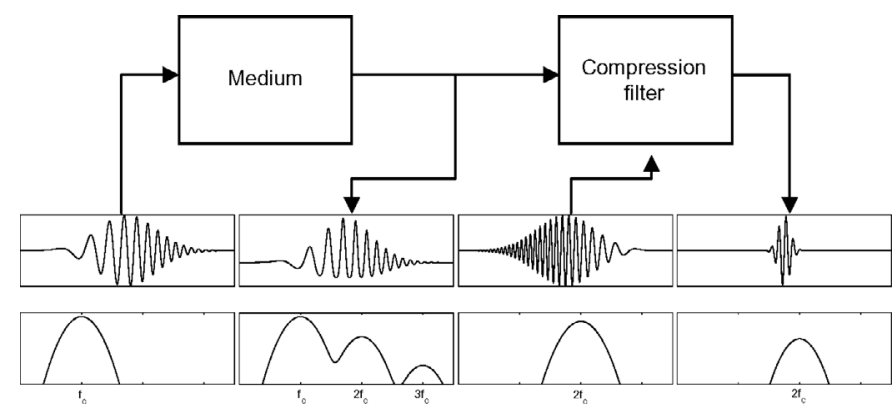

Fig. 2. Schematic of harmonic chirp compression method for selective compression and extraction of second harmonic part from received response. (Reprinted from Ultrasound in Medicine and Biology, J. Borsboom et al., "Nonlinear coded excitation method for ultrasound contrast imaging," vol. 29, pp. 277-284, (C) 2003, with permission from World Federation of Ultrasound in Medicine and Biology.)

sion of the contrast agent bubbles due to the longer length of the chirp and theoretically improves the CTR by the same amount.

In this paper we propose a chirp excitation method with a nonlinear decoder for use with contrast agent imaging. First, results from the nonlinear decoder on simulated bubble echoes will be examined. Second, the nonlinear chirp compression method is evaluated using measured reflections from a flat plate reflector that contain harmonics from nonlinear propagation. Third, in-vitro B-scan images using pulse and chirp excitations are compared to test the performance of the nonlinear compression technique invitro and to indicate the current limitations of this technique.

\section{THEORY}

Chirp excitation operates by transmission of a long frequency modulated burst into the medium. The main design parameters of a chirp are its length and its bandwidth, which determine the energy contained in the chirp and the obtainable axial resolution after compression, respectively. An additional apodization window can be used to lower the side-lobe levels that are inherent to compression. After reception of the echo signal, a compression filter removes the coding and recovers the axial resolution that was disturbed during the coding process. Conventionally, chirp compression takes a matched-filter approach in which an autocorrelation filter with an impulse response that is the time inverse of the transmitted chirp is used. As the compression filter has the same bandwidth as the fundamental of the transmitted chirp, this is equivalent to extracting the fundamental from the echo signal and adjusting the phase of the frequency components.

We propose a nonlinear compression filter that can selectively extract and compress the second harmonic from the received echo. Fig. 2 shows a schematic overview of the compression method. The first part is equal to the setup for regular chirp imaging; a chirp containing only the fundamental is sent into a nonlinear medium. The medium (the patient in clinical situations) generates an echo that 
contains both the fundamental and higher harmonics. Instead of using a matched filter based on the time inverse of the transmitted chirp as its impulse response, we defined a compression filter that, compared to a regular compression filter, has twice the instantaneous frequency at every time point. In addition, the apodization was changed to obtain the same bandwidth as we would get from frequency doubling the pulse that was used as excitation ${ }^{1}$. For example, if the excitation chirp ranges between 2 and $4 \mathrm{MHz}$, the compression filter will range between 4 and $8 \mathrm{MHz}$. In frequency domain this design is equivalent to a compression filter that extracts and compresses the second harmonic from the echo signal. The compressed echo has good axial resolution and a center frequency at the second harmonic of the transmitted chirp.

To compare regular pulse excitation, which uses short pulses with large bandwidth, and chirp excitation, which uses long bursts with large bandwidth, we designed a Gaussian envelope pulse and a Gaussian apodized chirp that have the same Fourier magnitude spectrum. Fig. 3 shows the pulse and the chirp in time and frequency domain that were used as excitation in the simulation study and the in-vitro validation. Center frequency and bandwidth of both pulses are $2 \mathrm{MHz}$ and $45 \%$, respectively. The $-6 \mathrm{~dB}$ length is $2.0 \mu \mathrm{s}$ for the pulse and $9.4 \mu \mathrm{s}$ for the chirp. When both pulses are scaled to equal peak amplitude, the energy difference between the pulse and the chirp is $10 \mathrm{~dB}$.

Previous research [11] has shown that the level of tissue harmonics due to nonlinear propagation, depends, other variables being equal, on peak pressure but not on pulse energy. Fig. 4 shows simulation results obtained from solving the KZK-equation, which models the effect of nonlinear propagation, diffraction and attenuation, in time domain for a focused 25 -mm diameter transducer with $75-\mathrm{mm}$ focal length using pulse and chirp having either equal peak pressure $(250 \mathrm{kPa})$ or equal signal energy $(250 \mathrm{kPa}$ pulse and $80 \mathrm{kPa}$ chirp). The curves show the simulated responses at focal depth. It is clear that a chirp produces significantly less harmonics than an equal energy pulse, and equal peak amplitude excitations produce relative levels of harmonics that are approximately equal. This effect is explained by the fact that generation of propagation harmonics only accumulates over propagation depth and not over pulse duration. The response from bubbles, however,

$$
\begin{aligned}
& { }^{1} \text { The actual chirp and compression filter impulse response used in } \\
& \text { this study were defined as: } \\
& \operatorname{chirp}(t)=e^{-\left(t-10 e^{-6}\right)^{2} / 2 \sigma^{2}} \cdot \cos \left(2 \pi \cdot\left(\frac{1.8 e^{6}}{20 e^{-6}} t+2.0 e^{6}\right) \cdot t\right) \\
& \text { filter }(t)=\left(e^{-\left(t-10 e^{-6}\right)^{2} / 2 \sigma^{2}}\right)^{4} \cdot \cos \left(2 \pi \cdot\left(\frac{-3.6 e^{6}}{20 e^{-6}} t+4.0 e^{6}\right) \cdot t\right)
\end{aligned}
$$

with

$$
\sigma=\frac{0.69}{2 e^{9} \cdot 2 \pi^{2}}
$$
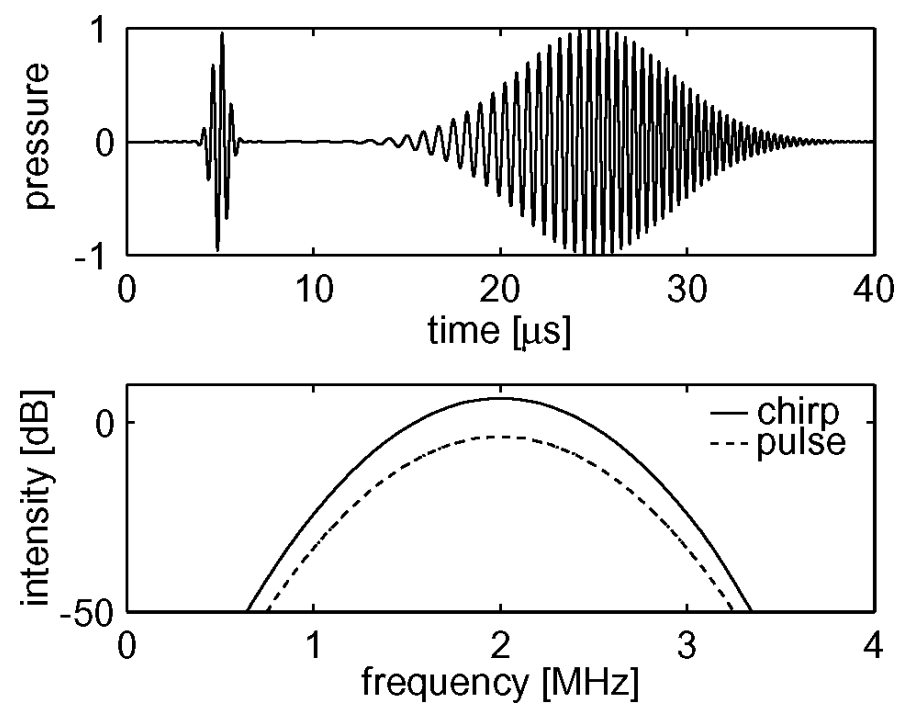

Fig. 3. Gaussian pulse and linear, Gaussian apodized chirp with equal center frequency, bandwidth, and equal Fourier magnitude spectra that were used as excitation in simulation study and experimental validation.

depends on both peak amplitude and pulse length. As bubble dynamics can be approximately described by a damped mass-spring system, effects of the excitation signal accumulate over pulse length, especially around the resonance frequency of the bubble.

For contrast agent bubbles, the generation of harmonics is mainly dependent on peak bubble wall excursion [12] Simulations have shown that chirp excitation causes larger bubble wall excursion than an equal amplitude pulse. Measurements on 2-D images obtained with an optical $25 \mathrm{MHz}$ fast framing camera system [13] are in agreement with the simulation results. Fig. 5 shows radius-time curves of a 3.8$\mu \mathrm{m}$ diameter contrast agent bubble recorded at 11.5 million frames per second as a function of time with the corresponding Fourier transform when subsequently excited with a pulse and a chirp with $2 \mathrm{MHz}$ center frequency and $45 \%$ bandwidth at approximately $120 \mathrm{kPa}$ peak pressure. Fig. 5 clearly shows that the peak bubble wall excursion is larger for chirp excitation than for pulse excitation. The Fourier transform clearly shows a difference in energy at the fundamental of $10 \mathrm{~dB}$. The second harmonic is visible for the chirp; however, poor SNR in the pulse response masked the second harmonic component.

To calculate the radius of a contrast agent bubble as a function of time when exposed to these excitation waveforms, we used a differential equation which is named after its developers Rayleigh, Plesset, Noltingk, Neppiras, and Poritski (RPNNP). The RPNNP equation, which describes the radial oscillations of an ideal gas bubble under time-varying acoustic pressure, was modified to include the effects of an additional restoring force due to shell stiffness and friction inside the shell [14]. A computer program was developed in Matlab (The Mathworks, Inc., Natick, MA) and $\mathrm{C}$ to solve the equation for a given excitation signal using a fifth order Runge-Kutta algorithm with variable step 

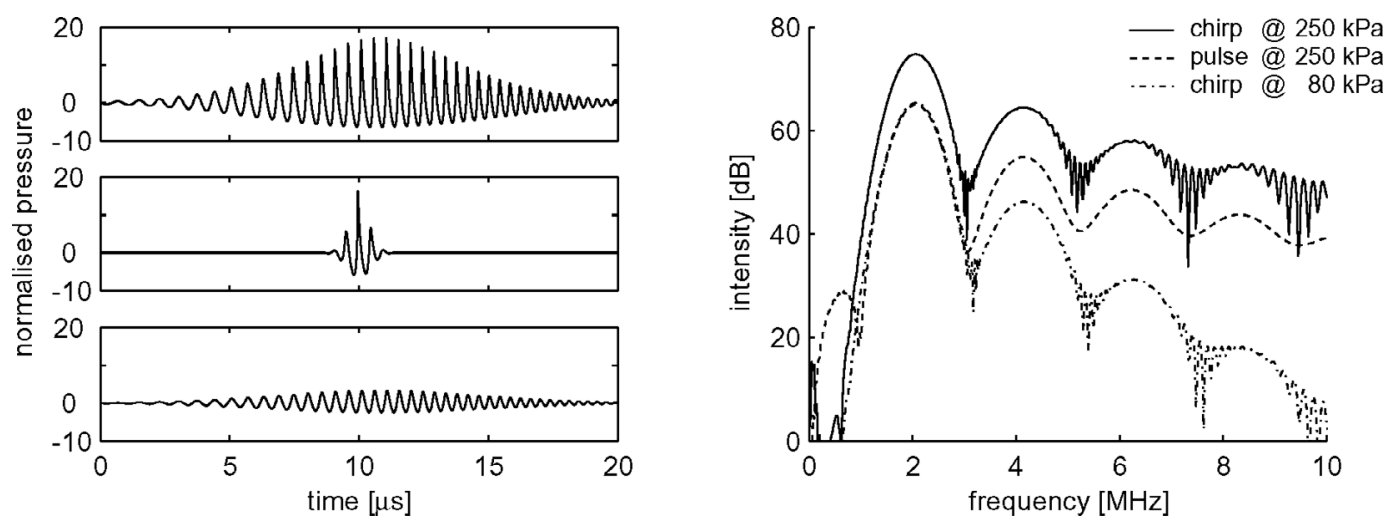

Fig. 4. Simulated nonlinear propagation from KZK-equation using pulse and chirp having either equal peak amplitude or equal energy in time (left) and frequency (right) domain. Equal peak amplitude excitations generate equal relative levels of harmonics, and equal energy chirp generates fewer harmonics than pulse.
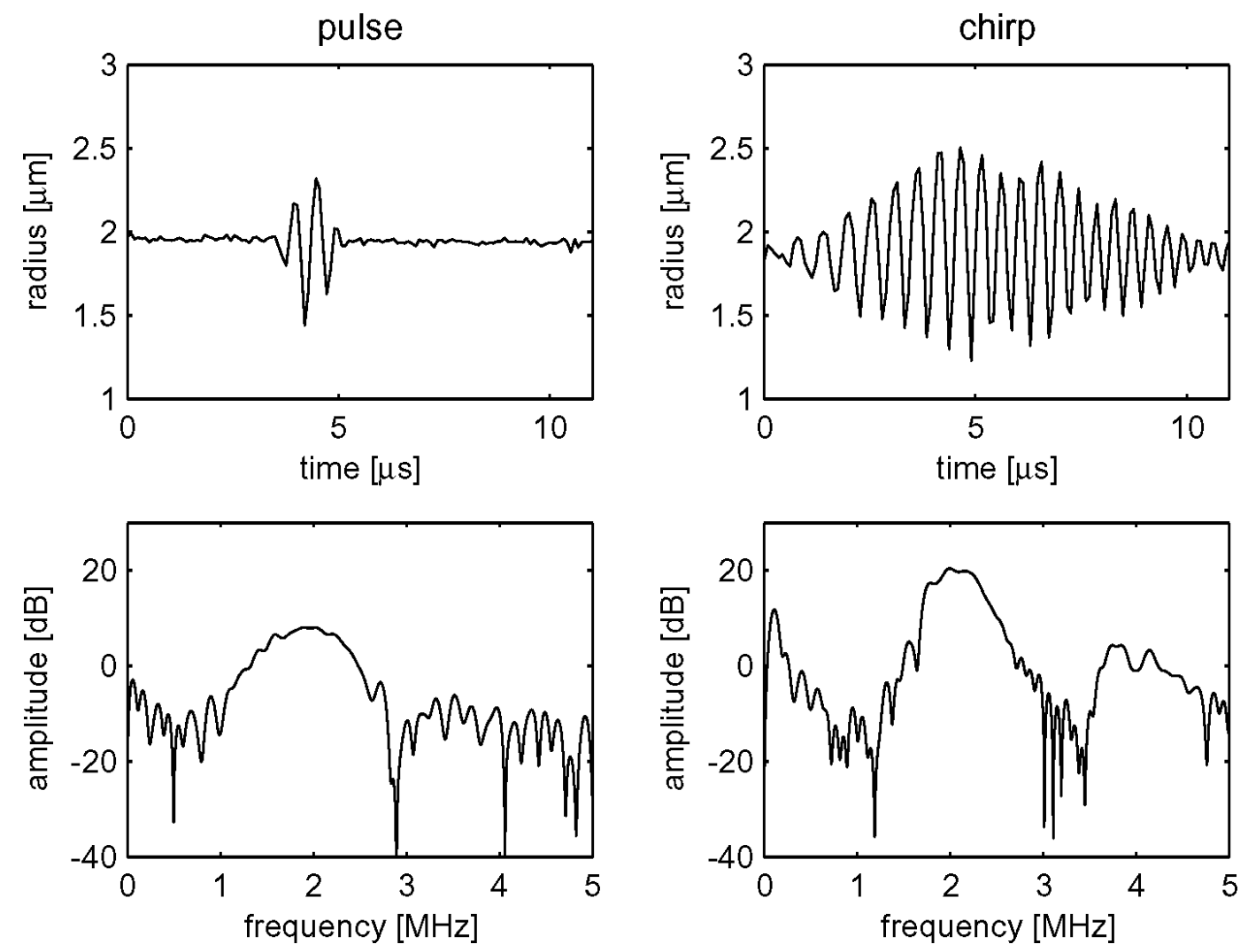

Fig. 5. Radial response of 3.8- $\mu \mathrm{m}$ diameter contrast agent bubble in time (top) and frequency (bottom) domain as observed with Brandaris128 fast framing camera at 11.5 million frames per second. The radial excursion is clearly larger for chirp (right) than for pulse (left).

size [15]. With the simulation program, we investigated the effect of different excitation signals on the generation of harmonics from contrast agent bubbles.

\section{METHOD}

The harmonic chirp imaging method, including the compression, was initially evaluated on simulated bubble echo signals and measured propagation harmonics. We simulated the echoes generated from $1.85 \mu \mathrm{m}$, $2.75 \mu \mathrm{m}$, and $4.50 \mu \mathrm{m}$ radius contrast agent bubbles with SonoVue ${ }^{\mathrm{TM}}$ parameters [16]. At these sizes the resonance frequencies of the bubbles are $3.5 \mathrm{MHz}, 2 \mathrm{MHz}$, and $1 \mathrm{MHz}$, respectively. Thus the bubbles are driven below, at and above their resonance frequencies for the given excitations. As excitation we used the $2-\mathrm{MHz}$ center frequency pulse and chirp as defined in Fig. 3 and scaled to $150 \mathrm{kPa}$ peak pressure.

In addition, we measured reflections from a flat steel reflector that was positioned at $75 \mathrm{~mm}$ from a $3.5 \mathrm{MHz}, 65 \%$ bandwidth unfocused, 12-mm diameter, single-element transducer (Imasonic, Besançon, France) in pulse-echo mode using the aforementioned pulse and chirp at approximately $1 \mathrm{MPa}$ peak pressure. Fig. 6 shows a schematic 


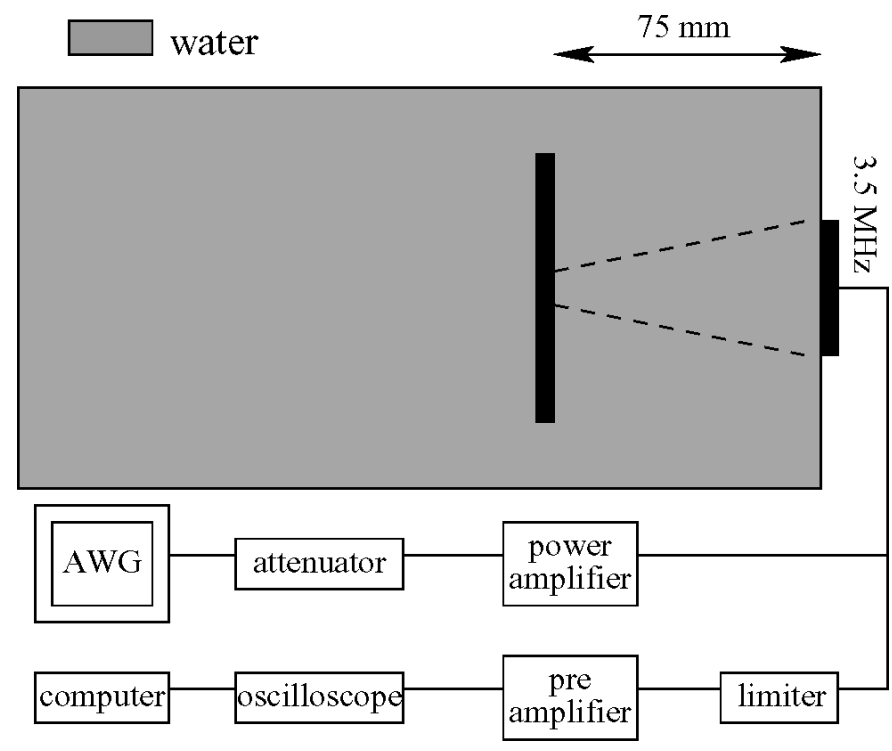

Fig. 6. Schematic setup of nonlinear propagation, pulse-echo measurement using an unfocused $3.5 \mathrm{MHz}, 65 \%$ bandwidth transducer and a steel plate reflector at $75 \mathrm{~mm}$ from the transducer.

setup for this measurement. The excitations were generated with an arbitrary waveform generator (LW420A, LeCroy, Chestnut Ridge, NY) and amplified by a $50 \mathrm{~dB}$ linear power amplifier (2100L, ENI, Rochester, NY). The amplitude was adjusted with a variable attenuator $(355 \mathrm{C} / \mathrm{D}$, Hewlett-Packard, Palo Alto, CA). The resulting echo was amplified and subsequently digitized and recorded with a LeCroy digital oscilloscope (9400A, LeCroy). The traces were transferred and processed off-line on an IBMcompatible computer. The simulated and measured echoes were either filtered in the case of pulse echo or compressed in the case of chirp echo to extract the fundamental and the second harmonic. The resulting signals were envelope detected and compared on the basis of axial resolution and side-lobe level.

To evaluate the performance of the nonlinear compression technique in-vitro, we obtained B-mode images by mechanically scanning over a flow phantom using the pulse and chirp excitations as defined in Fig. 3 in fundamental and second harmonic mode. The flow phantom consisted of $2 \%$ agar-agar gel with $0.4 \%$ carborundum particles to mimic tissue scattering. Embedded in the phantom were two flow channels of 10-mm and 5-mm diameter in which an experimental contrast agent (BR14, Bracco Research SA, Geneva, Switzerland) in 1:2,000 dilution was flowing. The flow channels were slightly angled relative to the incident ultrasound field to prevent specular reflections from the flow channel boundaries. The flow was maintained by a gear pump (GA-X21, Micropump Ltd., St. Neots, Cambridgeshire, UK) at approximately $0.6 \mathrm{ml} / \mathrm{s}$.

The phantom was mounted on a hand-operated $\mathrm{x}$ $\mathrm{y}$ table and translated relative to a fixed transducer to make the B-scan images. The transducer was the 3.5 MHz, single-element transducer. Both excitations were hydrophone calibrated to have equal peak amplitudes up to $\mathrm{MI}=0.2$. B-mode images were obtained by scanning the phantom by increments of $0.5 \mathrm{~mm}$ under the fixed transducer. The scanning direction was perpendicular to the direction of the flow. At each scanning position, the resulting echo was digitized and recorded with a LeCroy digital oscilloscope. The images were processed off-line on an IBM-compatible computer.

The performance of the nonlinear chirp compression technique was evaluated by comparing the apparent sizes of the flow channels in the chirp images to the sizes in the images made by conventional pulsed imaging. In addition, the tissue SNR levels are compared to quantify the differences between the two excitation methods. To obtain the tissue signal levels, appropriate regions in the images were selected and averaged. The noise level was determined from a separate noise measurement by using the entire system without generating an excitation signal.

\section{Results}

The envelope detected fundamental and second harmonic parts of the calculated bubble response and are shown in Fig. 7 for each bubble size excited with either pulse or chirp. The ordinates are shown on a log scale to visualize the side lobes from chirp compression. Apart from the differences in peak energy level of the main lobes in each figure, the main lobes look similar in shape. Table I shows the obtained axial resolution for each of the curves in Fig. 7. From Table I we see that the axial resolution is in all cases comparable, with only a very slight degradation for the bubble at resonance. However, the detected envelopes from chirp excitation exhibit range side lobes as was expected from the use of a compression filter. The side-lobe levels are all more than $50 \mathrm{~dB}$ below the main lobe.

Experimental results from the compression of chirp echoes from the flat plate are shown in Fig. 8. On logarithmic scale, Fig. 8 shows the envelope-detected signal using the linear and nonlinear compression filters on the echoes reflected by the flat plate generated by the pulse and chirp excitations. Table II quantifies axial resolutions for these measurements. We see that, as with the simulated bubble echoes, the curves from pulse and chirp look very similar. Axial resolutions, therefore, are comparable, which can be seen from Table II as well. Side-lobe levels can be seen to $60 \mathrm{~dB}$ and $30 \mathrm{~dB}$ below the main lobe for the linear and nonlinear compression filters, respectively.

Fig. 9 shows the B-mode images of the $10 \mathrm{~mm}$ and $5 \mathrm{~mm}$ flow channels embedded in the phantom as obtained with a single-element transducer in pulse-echo mode. The top row shows images from pulse and chirp in fundamental mode; the bottom row shows images in harmonic mode. The dynamic range in all images is $40 \mathrm{~dB}$, and all images are normalized to give good contrast between the flow channel and the surrounding tissue mimicking material. In all images the flow channel is clearly visible as an echogenic region. However, shadowing below the flow channels was visible; this may be caused by accumulation of contrast 

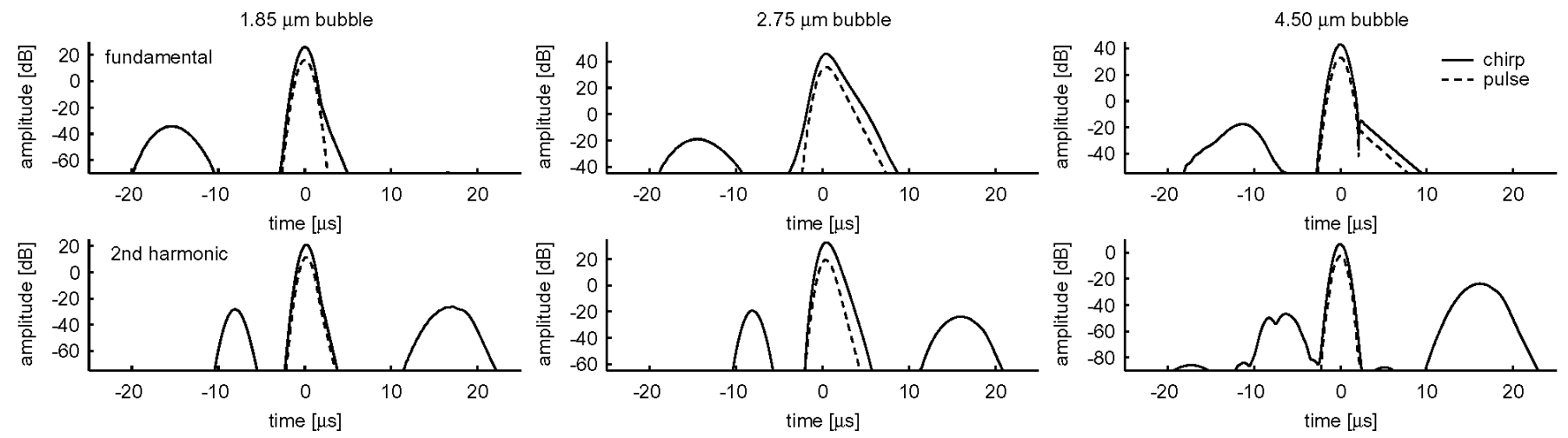

Fig. 7. Envelope detected fundamental and second harmonic responses of simulated $1.85 \mu \mathrm{m}, 2.75 \mu \mathrm{m}$, and $4.50 \mu \mathrm{m}$ radius bubbles excited with pulse and chirp.

TABLE I

Fundamental and Second Harmonic Axial Resolution (mm) Obtained for Simulated $1.85 \mu \mathrm{m}, 2.75 \mu \mathrm{M}$, and $4.50 \mu \mathrm{m}$ Radius Single Bubble Responses Using Pulse and Chirp Excitations.

\begin{tabular}{ccccccc}
\hline & \multicolumn{2}{c}{$1.85 \mu \mathrm{m}$} & \multicolumn{2}{c}{$2.75 \mu \mathrm{m}$} & \multicolumn{2}{c}{$4.50 \mu \mathrm{m}$} \\
\cline { 2 - 7 } & & 2nd & & 2nd & 2nd \\
& Fundamental & Harmonic & Fundamental & Harmonic & Fundamental & Harmonic \\
\hline Pulse & 2.1 & 1.8 & 2.7 & 2.1 & 2.0 & 1.7 \\
Chirp & 2.1 & 1.9 & 2.7 & 2.3 & 2.0 & 1.8 \\
\hline
\end{tabular}

TABLE II

Fundamental and Second Harmonic Axial Resolution (mm) for Flat Plate Reflector Using Pulse and Chirp Excitations.

\begin{tabular}{lcc}
\hline & Fundamental & 2nd Harmonic \\
\hline Pulse & 2.0 & 1.8 \\
Chirp & 2.2 & 1.9 \\
\hline
\end{tabular}

agent due to floatation. The apparent channel size agrees reasonably well between pulse and chirp excitation images in the same imaging mode. However, in the chirp harmonic images side lobes are visible as shadows above and below the flow channel. Speckle is well developed in the fundamental images, with the pulse images being slightly noisier due to the lower signal energy in the pulse excitation. The harmonic images suffer from relatively low SNR due to the low transmitted amplitude to decrease the generation of contaminating tissue harmonics. There is, however, some speckle visible through the noise for the chirp harmonic images, indicating the advantage of chirp excitation for harmonic images as well.

Table III shows tissue SNR and the sizes of the flow channels as measured from the images in the axial direction. The SNR value for pulse harmonic image could not be calculated as the image is too noisy to calculate a tissue signal. However, estimating this value from the SNR obtained from the chirp harmonic image, we estimate it to be lower than $0 \mathrm{~dB}$. An increase of approximately $10 \mathrm{~dB}$ in SNR was found for chirp excitation compared to pulse
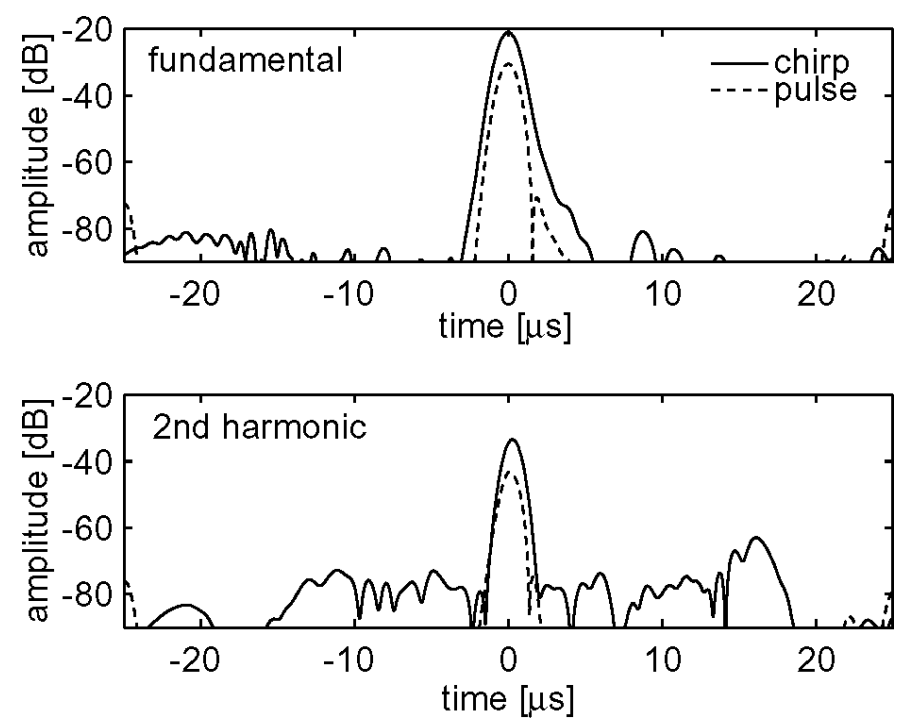

Fig. 8. Envelope detected fundamental and second harmonic of measured nonlinear propagation harmonics using pulse and chirp excitations.

excitation. The size of the flow channels is overestimated in all images. In fundamental mode, the imaged size of the flow channel agrees. For harmonic mode there is a slight increase in measured flow channel size for chirp.

\section{Discussion}

The use of chirp excitation signals is critically dependent on the performance of the compression filter to obtain 


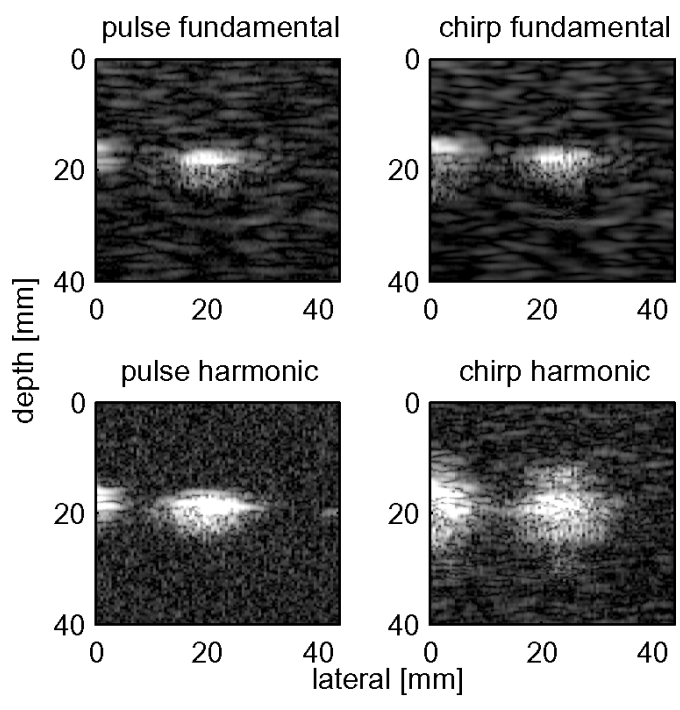

(a)

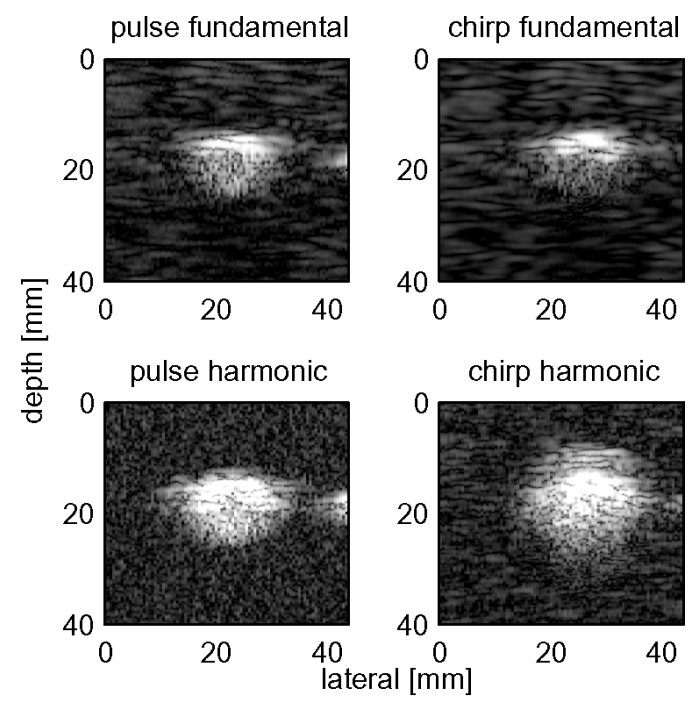

(b)

Fig. 9. In vitro B-mode images of flow phantom with embedded 5-mm (a) and 10-mm (b) flow channels containing contrast agent using pulse and chirp excitation displayed at $40 \mathrm{~dB}$ dynamic range.

TABLE III

SNR and Flow Channel Sizes from B-mode Images for Pulse and Chirp Excitations in Fundamental and Second Harmonic IMAging Mode.

\begin{tabular}{|c|c|c|c|c|}
\hline & \multicolumn{2}{|c|}{ Pulse } & \multicolumn{2}{|c|}{ Chirp } \\
\hline & Fundamental & $\begin{array}{c}\text { 2nd } \\
\text { Harmonic }\end{array}$ & Fundamental & $\begin{array}{c}\text { 2nd } \\
\text { Harmonic }\end{array}$ \\
\hline SNR & $19.3 \mathrm{~dB}$ & $<0 \mathrm{~dB}$ & $30.1 \mathrm{~dB}$ & $8.0 \mathrm{~dB}$ \\
\hline 5-mm channel & $8 \mathrm{~mm}$ & $9 \mathrm{~mm}$ & $8 \mathrm{~mm}$ & $10 \mathrm{~mm}$ \\
\hline 10-mm channel & $12 \mathrm{~mm}$ & $14 \mathrm{~mm}$ & $12 \mathrm{~mm}$ & $15 \mathrm{~mm}$ \\
\hline
\end{tabular}

a narrow main lobe and low-range, side-lobe levels. This becomes even more important when designing a compression filter for compression of harmonic signals. For good compression performance, the phase relations between the individual frequency components in the echo signal that is the input for the compression filter must be known when designing the compression filter. In a linear system this is the case as in those systems the change in phase relations is dependent only on the system itself, i.e., not on the phase relations in the signal that is input into the system. For the nonlinear case, this may not hold as frequency components are generated that are not in the input signal and, hence, may have arbitrary phase relations that prevent adequate compression.

The nonlinear compression filter has been shown to adequately compress simulated bubble echoes and measured propagation harmonics. Axial resolutions after compression are within $10 \%$ of the axial resolutions obtained from regular pulse excitation. Side-lobe levels are at least $30 \mathrm{~dB}$ below the main lobe for compressed, simulated, singlebubble responses and compressed propagation harmonics, except for the $4.50 \mu \mathrm{m}$ bubble in harmonic mode. Although these side-lobe levels may create visible ghost or blooming artefacts, they may be tolerable in cases in which sensitive detection of contrast agent is wanted.
By design, an increase of $10 \mathrm{~dB}$ in SNR for B-mode images obtained from chirp excitation over pulse excitation in both fundamental and harmonic mode was expected. This improvement is found in the chirp fundamental image and qualitatively observed in the chirp harmonic image, although the SNR for the pulse harmonic image cannot be quantified. Imaged size of the flow channel was found to be approximately equal when comparing the pulse fundamental images with the chirp fundamental images and $10 \%$ worse when comparing the chirp harmonic images with the pulse harmonic images. The CTR was expected to increase approximately $2 \mathrm{~dB}$ in the chirp harmonic images compared to the pulse harmonic images. In the images, however, the CTR was found to decrease $1 \mathrm{~dB}$ between chirp harmonic and pulse harmonic. This value was degraded by the fact that the tissue signal is lower than noise in the pulse harmonic image. Actual improvement in CTR can, hopefully, be demonstrated in future studies in which noise level is reduced by, for example, averaging. Therefore, we consider the 1-dB decrease in CTR a lower bound on the actual CTR difference in the images. Additionally, we clearly see that the speckle patterns of pulse fundamental images and chirp fundamental images are highly comparable, indicating a high similarity in the total response of imaging system and, therefore, axial resolution, 
although much different excitations are used. However, significant side lobes were seen in the chirp harmonic images. Although the side lobes at the top of the flow channel are probably exaggerated due to accumulation of contrast agent at the top of the flow channel observed during the experiments, this still indicates difficulties with either the matching of the compression filter to the transmitted chirp or a more fundamental difference in chirp harmonics generation for bubbles.

\section{Conclusions}

Chirp excitation with a nonlinear decoder has been shown to produce images with resolution comparable to pulse excitation. However, improvement in the suppression of compression artefacts (i.e., range side lobes) is needed.

\section{REFERENCES}

[1] B. A. Schrope and V. L. Newhouse, "Second harmonic ultrasonic blood perfusion measurement," Ultrasound Med. Biol., vol. 19, pp. 567-579, 1993.

[2] D. H. Simpson, C. T. Chin, and P. N. Burns, "Pulse inversion Doppler: A new method for detecting nonlinear echoes from microbubble contrast agents," IEEE Trans. Ultrason., Ferroelect., Freq. Contr., vol. 46, pp. 372-382, 1999.

[3] G. Brock-Fisher, M. Poland, P. Rafter, and M. Mooney, "Experimental observations of the sensitivity and frequency response of the power modulation technique for contrast imaging," in Proc. Fifth Heart Centre Eur. Symp. Ultrasound Contrast Imaging, 2000, pp. 1-77.

[4] M. I. Skolnik, Radar Handbook. New York: McGraw-Hill, 1970.

$[5]$ T. X. Misaridis, "Ultrasound imaging using coded signals," Ph.D. dissertation, Center for Fast Ultrasound Imaging, Ørsted, Technical University of Denmark, 2001.

[6] Y. Takeuchi, "Chirped excitation for $\leftarrow 100 \mathrm{~dB}$ time sidelobe echo sounding," in Proc. IEEE Ultrason. Symp., 1995, pp. 14331435.

[7] M. O'Donnell, "Coded excitation systems for improving the penetration of real-time phased-array imaging systems," IEEE Trans. Ultrason., Ferroelect., Freq. Contr., vol. 39, pp. 341-351, 1992.

[8] H. Becher and P. N. Burns, Handbook of Contrast Echocardiography: LV Function and Myocardial Perfusion. Berlin: SpringerVerlag, 2000.

[9] N. de Jong, "Mechanical index," Eur. J. Echocard., vol. 3, pp. 73-74, 2002.

[10] J. Borsboom, C. T. Chin, and N. de Jong, "Nonlinear coded excitation method for ultrasound contrast imaging," Ultrasound Med. Biol., vol. 29, pp. 277-284, 2003.

[11] Y. Lee and M. F. Hamilton, "Time-domain modelling of pulsed finite-amplitude sound beams," J. Acoust. Soc. Amer., vol. 97, pp. 906-917, 1995.

[12] J. Borsboom, C. T. Chin, and N. de Jong, "Experimental validation of a nonlinear coded excitation method for contrast agent imaging," in Proc. IEEE Ultrason. Symp., 2002, pp. 1933-1936.

[13] C. T. Chin, C. Lancée, J. Borsboom, F. Mastik, M. E. Frijlink, and N. de Jong, "Brandaris 128: A digital 25 million frames per second camera with 128 highly sensitive frames," Rev. Sci. Instrum., vol. 75, pp. 5026-5034, 2003.

[14] N. de Jong, R. Cornet, and C. T. Lancée, "Higher harmonics of vibrating gas filled microspheres. Part one: Simulations," Ultrasonics, vol. 32, pp. 447-453, 1994.

[15] W. H. Press, S. A. Teukolsky, W. T. Vetterling, and B. P. Flannery, Numerical Recipes in C: The Art of Scientific Computing. Cambridge: Cambridge Univ. Press, 1993.

[16] J.-M. Gorce, M. Arditi, and M. Schneider, "Influence of bubble size distribution on the echogenicity of ultrasound contrast agents: A study of SonoVue ${ }^{\mathrm{TM}}$," Invest. Radiol., vol. 35, pp. 661-671, 2000.

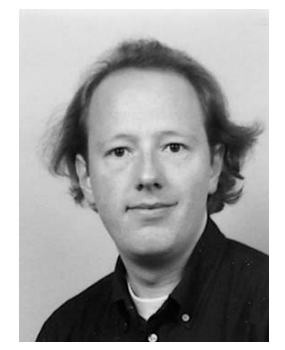

Jerome Borsboom was born in the Netherlands in 1975. He earned a M.Sc. degree in electrical engineering at Delft University of Technology, the Netherlands, in 1997. Since then, he has been working towards a degree in psychology at Leiden University, the Netherlands. In May 2000, he joined the department of Experimental Echocardiography of the thoraxcentre, Erasmus MC in Rotterdam, the Netherlands to pursue his Ph.D. under guidance of Nico de Jong. His research interests include contrast agent detection methods and open source software development.

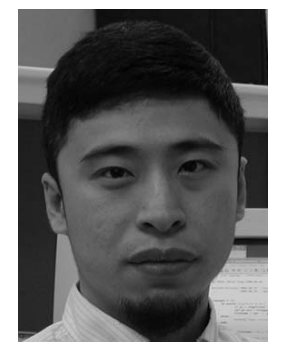

Chien Ting Chin was born in Hong Kong in 1969. He earned a B.A.Sc. in engineering science at the University of Toronto in 1993 and a M.Sc. degree on short-wavelength lasers in 1995. He obtained a Ph.D. degree in medical biophysics at the University of Toronto in 2001 , based on his theoretical and experimental work on ultrasound contrast agents. From 2001-2003 he worked at the Erasmus University Medical Centre in Rotterdam on a high speed optical imaging system for acoustic microbubbles. He currently works at Philips Research at Briarcliff Manor, New York, on diagnostic and other applications of microbubble agents.

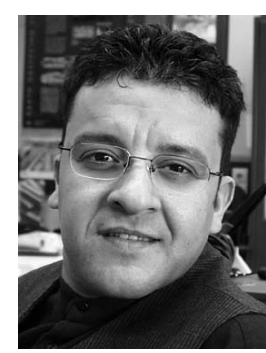

Ayache Bouakaz graduated from the University of Stif, Algeria, from the Dept. of Electrical Engineering. He obtained his Ph.D. in 1996 from the Dept. of Electrical Engineering at the University of ISNA, Lyon, France. In 1998 he joined the Dept. of Bioengineering at PennState University in State College, PA, USA, where he worked as a postdoc for one year. Since February 1999, he is employed at the Erasmus University Medical Ctr., Rotterdam, The Netherlands. His research focuses on imaging, ultrasound contrast agents and

transducer design.

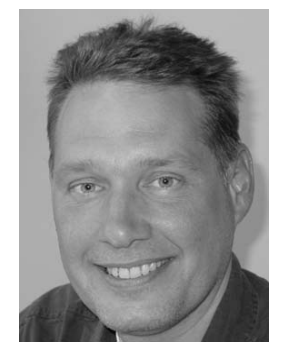

Michel Versluis was born in the Netherlands in 1963. He graduated in Physics in 1988 at the University of Nijmegen, the Netherlands, with a special interest in Molecular Physics and Astrophysics, working in the field of far-infrared laser spectroscopy of interstellar molecular species. Later, he specialized in the application of intense tunable UV lasers for flame diagnostics resulting in a successful defense of his $\mathrm{PhD}$ thesis in 1992. After a two year research position working on molecular dynamics at Griffith University, Brisbane, Australia he continued to work on developing laser diagnostic techniques for internal combustion engines (Lund, Sweden) and industrial jet flames and solid rocket propellants (Delft, The Netherlands). Michel Versluis is now a lecturer at the University of Twente, the Netherlands in the Physics of Fluids group working on the experimental study of bubbles and jets in multiphase flows and granular flows. He also works on the use of microbubbles as tools for medical diagnosis and therapy. 


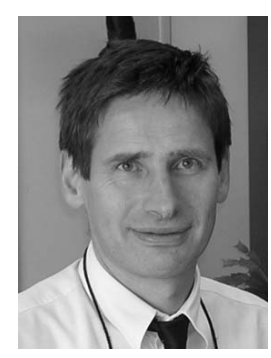

Nico de Jong (A'97) was born in 1954. He graduated from Delft University of Technology, The Netherlands, in 1978. He got his M.Sc. in the field of pattern recognition. Since 1980, he has been a staff member of the Thoraxcenter of the Erasmus University Medical Ctr., Rotterdam, The Netherlands. At the Dept. of Biomedical Engineering, he developed linear and phased array ultrasonic probes for medical diagnosis, especially compound and transesophageal transducers. In
1986 his interest in ultrasound applications shifted toward the theoretical and practical background of ultrasound contrast agents. In 1993 he received his Ph.D. for "Acoustic properties of ultrasound contrast agents." Currently he is interested in the development of 3 -D transducers and a fast framing camera system. De Jong is the project leader of STW and FOM projects on ultrasound contrast imaging and drug delivery systems. Together with Folkert ten Cate, $\mathrm{MD}$, he is organizer of the annual European Symposium on Ultrasound Contrast Imaging, held in Rotterdam and attended by approximately 175 scientists from all over the world. 